\title{
Phantom Field from Conformal Invariance
}

\author{
Mokhtar Hassaïne* \\ Instituto de Matemática y Física, Universidad de Talca, Casilla 747, Talca, Chile, and \\ Centro de Estudios Científicos (CECS), Casilla 1469, Valdivia, Chile.
}

\begin{abstract}
We establish a correspondence between a conformally invariant complex scalar field action (with a conformal self-interaction potential) and the action of a phantom scalar field minimally coupled to gravity (with a cosmological constant). In this correspondence, the module of the complex scalar field is used to relate conformally the metrics of both systems while its phase is identified with the phantom scalar field. At the level of the equations, the correspondence allows to map solution of the conformally non-linear Klein-Gordon equation with vanishing energy-momentum tensor to solution of a phantom scalar field minimally coupled to gravity with cosmological constant satisfying a massless Klein-Gordon equation. The converse is also valid with the advantage that it offers more possibilities owing to the freedom of rewriting a metric as the conformal transformation of another metric. Finally, we provide some examples of this correspondence.
\end{abstract}

\section{INTRODUCTION}

Conformal techniques and conformal invariance have been widely used in general relativity, quantum field theory in curved spaces and in string theories (for a review, see e.g. [1]). It is well-known that the massless KleinGordon action for a scalar field is conformally invariant only in two dimensions while in higher dimensions, the conformal invariance can be achieved by introducing a nonminimal coupling 2]. In this paper, we precisely consider the conformally invariant Klein-Gordon action for a complex scalar field $\Psi$ in arbitrary dimension with a potential term that does not spoil the conformal invariance. We restrict ourselves to a particular class of conformal transformation for which the conformal factor is given by a power of the module of the complex scalar field. For a particular value of the exponent, we prove that the conformally invariant Klein-Gordon action rescales to the Einstein-Hilbert action with a cosmological constant and with a source given by a phantom field. The terminology of phantom field refers to the fact that its kinetic energy term enters in the action with the opposite sign. As shown below, this is a direct consequence of the fact that the phantom field is described by the phase of the complex scalar field. In the recent literature, phantom field has been proposed to model the observational evidence for accelerating universe, [3] and [4]. In this case, the resulting equation of state parameter is given by $\omega=p / \rho<-1$ where $p$ is the pressure and $\rho$ the energy density. In contrast, the usual scalar field theories used to model the dark energy as the quintessence or the tachyonic scalar fields lead to an equation of state $\omega \geq-1$ which results as a consequence of imposing the dominant energy condition. However, classical tests of cosmology do not seem to exclude the regime $\omega<-1$, [3].

The correspondence between conformally invariant

*Electronic address: hassaine-at-inst-mat.utalca.cl complex scalar field and the phantom field coupled to gravity is also established at the level of the equations of motion. More precisely, solutions of the non-linear Klein-Gordon equation together with the vanishing of the energy-momentum tensor are mapped to solutions of a phantom scalar field satisfying a massless Klein-Gordon equation and minimally coupled to gravity with a cosmological constant. The converse of this mapping is also valid with the advantage that it offers more possibilities reflected by the freedom of rewriting a metric as the conformal of another metric. In particular, considering the metric as the conformal of itself, the same metric can described the spacetime geometry of both systems.

The clue of this correspondence lies in the conformal invariance of the generalized Klein-Gordon action. Hence its coupling with standard Einstein gravity will break the conformal invariance. However, in three dimensions gravity governed by the Cotton tensor enjoys the conformal invariance and in this case, the correspondence is established with the topologically massive gravity [5] with a cosmological constant and with a phantom source.

The plan of the paper is organized as follows. We review the conformally invariant Klein-Gordon action for a complex scalar field with a self-interacting potential. We explicitly show that this action rescales to the action of a phantom scalar field minimally coupled to gravity with a cosmological constant. This correspondence is also established at the level of the equations of motion. A particular attention is devoted to the three-dimensional case for which the conformally invariant Klein-Gordon action is interpreted as the source of conformal gravity governed by the Cotton tensor. In this case, the system is put in equivalence with topologically massive gravity with a cosmological constant and with a phantom source. We present some examples of this correspondence. Finally, an appendix is given in order to explicitly prove the correspondence at the level of the actions as well their corresponding equations of motion. 


\section{PHANTOM FROM CONFORMAL INVARIANCE}

We consider the conformally invariant Klein-Gordon action for a complex scalar field in $D$ dimensions,

$$
\begin{array}{r}
I=\int d^{D} x \sqrt{-g}\left(\frac{1}{2}\left(\partial_{\alpha} \Psi\right)\left(\partial^{\alpha} \Psi^{\star}\right)+\frac{\xi_{D}}{2} R \Psi \Psi^{\star}\right. \\
\left.+\lambda\left(\Psi \Psi^{\star}\right)^{\frac{D}{D-2}}\right),
\end{array}
$$

where $R$ represents the scalar curvature of the metric, $\lambda$ is the potential strength and $\xi_{D}$ is the conformal nonminimal coupling parameter given by

$$
\xi_{D}=\frac{(D-2)}{4(D-1)} \text {. }
$$

It is well-known that for this specific value (2), the action (11) possesses a conformal invariance for which the implementation on the dynamical fields is given by

$$
g_{\mu \nu} \rightarrow \Omega^{2} g_{\mu \nu}, \quad \Psi \rightarrow \Omega^{\frac{2-D}{2}} \Psi
$$

The equation of motion obtained by varying this action with respect to the scalar field yields to a non-linear generalized Klein-Gordon equation

$$
\square \Psi=\xi_{D} R \Psi+\frac{2 \lambda D}{(D-2)}\left(\Psi \Psi^{\star}\right)^{\frac{2}{D-2}} \Psi .
$$

The variation of (1) with respect to the metric implies the vanishing of the energy-momentum tensor, i. e.

$$
\begin{aligned}
& T_{\mu \nu}:=\nabla_{(\mu} \Psi \nabla_{\nu)} \Psi^{\star}+\xi_{D}\left(g_{\mu \nu} \square-\nabla_{\mu} \nabla_{\nu}+G_{\mu \nu}\right)|\Psi|^{2} \\
& -g_{\mu \nu}\left(\frac{1}{2} \nabla_{\sigma} \Psi \nabla^{\sigma} \Psi^{\star}+\lambda\left(\Psi \Psi^{\star}\right)^{\frac{D}{D-2}}\right)=0
\end{aligned}
$$

For later convenience, we decompose the complex scalar field into its module and phase as $\Psi=\sqrt{\rho} e^{i \theta}$.

We now show that, under a particular conformal transformation, the action (11) rescales to the action of a phantom scalar field coupled to gravity with a cosmological constant. Indeed, it is straightforward to see that in the conformal frame given by

$$
g_{\mu \nu}^{\prime}=\rho^{\frac{2}{D-2}} g_{\mu \nu}
$$

the scalar field action (11) becomes, up to a boundary term,

$$
I^{\prime}=\frac{\xi_{D}}{2} \int d^{D} x \sqrt{-g^{\prime}}\left[R^{\prime}-2\left(-\frac{\lambda}{\xi_{D}}\right)+\frac{1}{\xi_{D}} \partial_{\mu} \theta \partial^{\mu} \theta\right] .
$$

We recognize the action of a phantom scalar field minimally coupled to gravity with a cosmological constant. We refer to the dynamical field $\theta$ as a phantom scalar field since its kinetic term enters in the action (7) with the opposite sign (we are using the mostly plus signature). In the expression (7), the cosmological constant $\Lambda$ is expressed in terms of the potential strength and the conformal nonminimal coupling parameter (2) as

$$
\Lambda=-\frac{\lambda}{\xi_{D}}=-\frac{4 \lambda(D-1)}{(D-2)} \text {. }
$$

The field equations associated to the rescaled action (7) read

$$
\begin{array}{r}
G_{\mu \nu}^{\prime}-\frac{\lambda}{\xi_{D}} g_{\mu \nu}^{\prime}=\frac{1}{\xi_{D}} \vartheta_{\mu \nu}, \\
\square^{\prime} \theta=0,
\end{array}
$$

where the energy-momentum tensor $\vartheta_{\mu \nu}$ is given by

$$
\vartheta_{\mu \nu}=-\partial_{\mu} \theta \partial_{\nu} \theta+\frac{1}{2} g_{\mu \nu}^{\prime} g^{\prime \alpha \beta} \partial_{\alpha} \theta \partial_{\beta} \theta
$$

The correspondence is also valid at the level of the equations of motion. Indeed, the energy-momentum tensor $T_{\mu \nu}$ defined in Eq. (5) becomes in the new frame (6)

$$
\begin{array}{r}
T_{\mu \nu}=\xi_{D} \rho\left[G_{\mu \nu}^{\prime}-\frac{\lambda}{\xi_{D}} g_{\mu \nu}^{\prime}-\frac{1}{\xi_{D}}\left(-\partial_{\mu} \theta \partial_{\nu} \theta\right.\right. \\
\left.\left.+\frac{1}{2} g_{\mu \nu}^{\prime} g^{\prime \alpha \beta} \partial_{\alpha} \theta \partial_{\beta} \theta\right)\right] .
\end{array}
$$

Hence, the vanishing of the energy-momentum tensor (5) is equivalent to the Einstein equations with phantom source (9a). It can also be shown that the imaginary part of the non-linear Klein-Gordon equation (4) becomes the massless Klein-Gordon equation for the phantom field (9b) while its real part implies

$$
R^{\prime}=\frac{2 \lambda D}{(2-D) \xi_{D}}-\frac{1}{\xi_{D}} \partial_{\sigma} \theta \partial^{\sigma} \theta
$$

which is a consequence of the Einstein equations (9a).

In sum, we have shown that starting from a complex scalar field $\Psi=\sqrt{\rho} e^{i \theta}$ and a metric $g_{\mu \nu}$ satisfying the equations (45), the rescaled metric (6) together with the phase $\theta$ are solutions of the Einstein equations with phantom source (9). The converse is also valid with the advantage that it offers more possibilities of mapping. Indeed, there exists a freedom of rewriting the metric solution $g_{\mu \nu}^{\prime}$ as the conformal of another metric and each of these choices will yield to a different solution of the Klein-Gordon action by engineering inverse. In particular, the same metric can described the spacetime geometry of both system provided the complex scalar field is only giving by a phase term, $\Psi=e^{i \theta}$.

\section{A. Three-dimensional conformal gravity}

The clue of this correspondence is due to the conformal invariance of the Klein-Gordon action (11) and, hence the coupling of this action to the Einstein gravity will explicitly break this invariance. In three dimensions, the 
action (11) can be seen as the source of conformal gravity governed by the Cotton tensor and consequently the full system enjoys the conformal invariance. In this case, the three-dimensional field equations become

$$
\begin{array}{r}
\square \Psi=\frac{1}{8} R \Psi+6|\Psi|^{2} \Psi, \\
C_{\mu \nu}=\kappa T_{\mu \nu},
\end{array}
$$

where the energy-momentum tensor $T_{\mu \nu}$ is given by the expression (51) with $D=3$ and $C_{\mu \nu}$ is the Cotton tensor defined by 12 ]

$$
C^{\mu \nu}=\frac{1}{\sqrt{-g}} \epsilon^{\mu \alpha \beta} D_{\alpha}\left(R_{\beta}^{\nu}-\frac{1}{4} \delta_{\beta}^{\nu} R\right) .
$$

This tensor is symmetric, identically conserved and traceless. Hence, since the Cotton tensor enjoys the conformal symmetry, it is easy to see that in the frame

$$
g_{\mu \nu}^{\prime}=\rho^{2} g_{\mu \nu},
$$

the equations of three-dimensional conformal gravity with source (13) become a massless Klein-Gordon equation for the phantom field, i.e. $\square^{\prime} \theta=0$, together with

$\frac{1}{\mu} C_{\mu \nu}^{\prime}+G_{\mu \nu}^{\prime}-8 \lambda g_{\mu \nu}^{\prime}=8\left[-\partial_{\mu} \theta \partial_{\nu} \theta+\frac{1}{2} g_{\mu \nu}^{\prime} \partial_{\sigma} \theta \partial^{\sigma} \theta\right](15)$

where $\mu=-\kappa / 8$. We recognize the equations describing a phantom scalar field acting as a source for topologically massive gravity with cosmological constant. To be more precise, in order to make contact with topologically massive gravity the signs of the topological mass $\mu$ and the dimensionless parameter $\kappa$ must be different. This ambiguity is irrelevant when the constants of the problem are not tied since we are just mapping solution of a system to solution of another one.

\section{B. Examples}

In what follows, we provide three explicit examples of the correspondence. The first two ones are given by the Siklos spacetime which provides a natural setup of the machinery described before since this spacetime is conformally related to a pp wave geometry. The third example is an ultra static wormhole solution of the Einstein equations with a phantom source.

\section{The pp wave metric and the Siklos spacetime}

We propose to solve the Einstein equations with a phantom source (9) in $D$ dimensions with a geometry given by

$$
\begin{array}{r}
d s^{2}=\frac{(D-2)^{2}}{8 \lambda y^{2}}\left[-F(u, y) d u^{2}-2 d u d v+d y^{2}\right. \\
\left.+d x_{1}^{2}+\cdots+d x_{D-3}^{2}\right] .
\end{array}
$$

This metric belongs to the class of Siklos spacetimes which are the only non-trivial Einstein spaces conformal to non-flat pp waves. These metrics are of importance in the study of propagation of gravitational waves in presence of a cosmological constant. Recently, real scalar fields nonminimally coupled to this geometry have been derived in three dimensions [9].

We assume that the null Killing field $k^{\mu} \partial_{\mu}=\partial_{v}$ is also a symmetry of the phantom scalar field, that means $\theta=\theta(u, y, \vec{x})$. In this case, it is easy to see that the only non-vanishing component of the Einstein tensor with cosmological constant is the one along the retarded time (uu) that reads

$$
G_{u u}-\frac{\lambda}{\xi_{D}} g_{u u}=\frac{1}{2 y}\left(\left(\partial_{y y} F\right) y-(D-2) \partial_{y} F\right) .
$$

Moreover, the remaining Einstein equations reduce to the vanishing of the energy-momentum tensor $\vartheta_{\mu \nu}$ which in turn imply that the function $\theta$ depends only on the retarded time, $\theta=\theta(u)$. Finally, the integration of the $u u$-component of the Einstein equations yields for $D \neq 3$

$$
F(u, y)=\frac{1}{\xi_{D}(D-3)}\left(\partial_{u} \theta\right)^{2} y^{2}+C(u) y^{D-1},
$$

while in three dimensions the structural function is given by

$$
F(u, y)=-8\left(\partial_{u} \theta\right)^{2} y^{2} \ln y+4\left(\partial_{u} \theta\right)^{2} y^{2}+C(u) y^{2} .
$$

In both cases, the real scalar field $\theta$ is an arbitrary function of the retarded time and $C$ is an undetermined integration function of the retarded time.

By engineering inverse, we conclude that the following complex scalar field

$$
\Psi(u, y)=\left[\frac{(D-2)}{8 \lambda y^{2}}\right]^{\frac{D-2}{4}} e^{i \theta(u)},
$$

together with the pp wave metric

$$
d s^{2}=-F(u, y) d u^{2}-2 d u d v+d y^{2}+\sum_{i=1}^{D-3} d x_{i}^{2},
$$

satisfy the non-linear Klein-Gordon equation with vanishing energy-momentum tensor (45).

\section{Conformal gravity in three dimensions with Siklos spacetime}

We now consider the equations of topologically massive gravity with cosmological constant with a source given by a phantom scalar field (15). As previously, we look for a geometry whose line element is given by the Siklos spacetime (16),

$$
d s^{2}=\frac{1}{8 \lambda y^{2}}\left[-F(u, y) d u^{2}-2 d u d v+d y^{2}\right] .
$$


In this case, the only non-vanishing component of the left hand side of the equations (15) is the one along the retarded time $u u$. As previously, the vanishing of the remaining component of the energy-momentum tensor implies that the scalar field $\theta$ depends only on the retarded time $u$, and hence the component $u u$ of the equations (15) becomes

$-\frac{8 \sqrt{2 \lambda}}{\kappa} y \partial_{y y y} F+\frac{1}{2 y^{2}}\left(y^{2} \partial_{y y} F-y \partial_{y} F\right)=-8\left(\partial_{u} \theta\right)^{2}$.

For a coupling constant $2 \lambda \neq(\kappa / 16)^{2}$, the integration of this equation yields

$$
\begin{aligned}
F(u, y) & =C_{1}(u) y^{2}+C_{2}(u) y^{\frac{\kappa \sqrt{2}}{32 \sqrt{\lambda}}+1} \\
& -\frac{8 \kappa y^{2} \ln y\left(\partial_{u} \theta\right)^{2}}{(\kappa-16 \sqrt{2 \lambda})}+\frac{4 \kappa y^{2}\left(\partial_{u} \theta\right)^{2}}{(\kappa-16 \sqrt{2 \lambda})^{2}}(\kappa-48 \sqrt{2 \lambda})
\end{aligned}
$$

where $C_{1}$ and $C_{2}$ are two undetermined functions of the retarded time. For the special coupling $2 \lambda=(\kappa / 16)^{2}$, the solution is

$$
F(u, y)=C_{1}(u) y^{2}+C_{2}(u) y^{2} \ln y+4\left(\partial_{u} \theta\right)^{2} y^{2}(\ln y)^{2} .
$$

Hence, using the correspondence one concludes that for a coupling constant $2 \lambda \neq(\kappa / 16)^{2}$, the complex scalar field given by

$$
\Psi=\frac{1}{\sqrt{\sqrt{8 \lambda} y}} e^{i \theta(u)},
$$

together with the pp wave geometry for which the metric function $F(u, y)$ is given by (22) are solutions to the three-dimensional conformal gravity equations (13). For the special coupling $2 \lambda=(\kappa / 16)^{2}$, the metric function of the pp wave geometry is given by (23) and the complex scalar field is

$$
\Psi=\sqrt{\frac{8}{\kappa y}} e^{i \theta(u)},
$$

where $\theta$ is an arbitrary function of the retarded time.

\section{Massless wormholes in four dimensions}

In four dimensions, there exists a regular ultra static wormhole solution of the Einstein equations without cosmological constant, i.e. equations (9) with $\lambda=0$. The metric solution in isotropic coordinates reads [10]-11],

$$
d s^{2}=-d t^{2}+\frac{c^{2}}{4}\left(1+\frac{1}{r^{2}}\right)^{2}\left(d x^{2}+d y^{2}+d z^{2}\right),
$$

with $r^{2}=x^{2}+y^{2}+z^{2}$ and, the phantom scalar field $\theta$ is given by

$$
\theta=\frac{\sqrt{3}}{3} \arctan \left(\frac{r^{2}-1}{2 r}\right) .
$$

The spatial sections have the form of an Einstein-Rosen bridge joining two isometric regions each with vanishing ADM mass. The wormhole metric (25) can be seen as the conformal of itself and, hence the complex scalar field defined by

$$
\psi=e^{i \frac{\sqrt{3}}{3} \arctan \left(\frac{r^{2}-1}{2 r}\right)},
$$

with the wormhole metric (25) are also solutions of the original equations (45) without self-interacting potential $\lambda=0$.

\section{DISCUSSION}

Here, we have shown that a phantom field coupled to gravity can be put in equivalence with a conformally invariant complex scalar field. The spacetime geometry of both systems are conformally related with a factor proportional to a power of the module of the complex scalar field while its phase plays the role of the phantom field. This last fact legitimates the reason for which the phantom kinetic term enters in the action with the opposite sign. Indeed, being purely imaginary its kinetic expression arises with a negative sign. In the current literature, the phantom field and its peculiar dynamics have been introduced to model the observed acceleration of the scale factor of the universe. In view of the correspondence reported here, an interesting and natural work will consist to analyze the cosmological consequences of interpreting the phantom field as a conformally invariant complex scalar field.

We have also established that the correspondence allows to map solutions of the conformally action to those of the phantom field minimally coupled to gravity. The converse is also valid with the advantage that it offers more possibilities of mapping. For a metric and a phantom field satisfying the Einstein equations with a massless Klein-Gordon equation, one can generate a wide variety of solutions of the non-linear Klein-Gordon equation with vanishing energy-momentum tensor. All these possibilities are due to the freedom of rewriting a metric as the conformal of another metric, and each of these choices will yield to a different solution of the non-linear Klein-Gordon equation with $T_{\mu \nu}=0$. However, in all these cases the complex scalar field solutions will have the same phase and, in some sense they will belong to a same class of equivalence. In particular, it is intriguing that the same metric can described both systems provided that the complex scalar field is only given by a phase term.

Finally, to conclude it would be desirable to have a physical interpretation of this correspondence.

Acknowledgments.- We thank E. Ayón-Beato, J. Gomis, C. Martínez, R. Troncoso and J. Zanelli for useful discussions. This work is partially supported by grants 1051084 from FONDECYT. Institutional support to the Centro de Estudios Científicos (CECS) from Empresas 
CMPC is gratefully acknowledged. CECS is a Millennium Science Institute and is funded in part by grants from Fundación Andes and the Tinker Foundation.

\section{APPENDIX A: CONFORMAL TRANSFORMATIONS}

We consider a conformal transformation of the metric $g_{\mu \nu}$ as

$$
g_{\mu \nu}^{\prime}=\rho^{\frac{2}{D-2}} g_{\mu \nu}, \quad \text { or } \quad g_{\mu \nu}=\rho^{\frac{2}{2-D}} g_{\mu \nu}^{\prime}
$$

Under this transformation, the operator $\square=\nabla_{\mu} \nabla^{\mu}$ becomes

$$
\begin{gathered}
\square^{\prime}=\frac{1}{\rho^{\frac{2}{D-2}}} \square+\frac{1}{\rho^{\frac{D}{D-2}}} g^{\alpha \beta}\left(\partial_{\alpha} \rho\right) \partial_{\beta}, \\
\square=\rho^{\frac{2}{D-2}}\left[\square^{\prime}-\frac{1}{\rho} g^{\prime \alpha \beta}\left(\partial_{\alpha} \rho\right) \partial_{\beta}\right] .
\end{gathered}
$$

The scalar curvature becomes

$$
\begin{aligned}
& R^{\prime}=\frac{1}{\rho^{\frac{2}{D-2}}}\left[R+\frac{(D-1)}{(D-2)}\left(-2 \frac{\square \rho}{\rho}+\frac{g^{\alpha \beta} \nabla_{\alpha} \rho \nabla_{\beta} \rho}{\rho^{2}}\right)\right] \\
& R=\rho^{\frac{2}{D-2}}\left[R^{\prime}+\frac{(D-1)}{(D-2)}\left(\frac{2 \square \rho}{\rho}-3 \frac{g^{\prime \alpha \beta} \nabla_{\alpha} \rho \nabla_{\beta} \rho}{\rho^{2}}\right)\right]
\end{aligned}
$$

[1] V. Faraoni, E. Gunzig and P. Nardone, Fund. Cosmic Phys. 20, 121 (1999).

[2] C. G. . Callan, S. R. Coleman and R. Jackiw, Annals Phys. 59, 42 (1970);

[3] R. R. Caldwell, Phys. Lett. B 545, 23 (2002)

[4] S. M. Carroll, M. Hoffman and M. Trodden, Phys. Rev. D 68, 023509 (2003)

[5] S. Deser, R. Jackiw and S. Templeton, Annals Phys. 140, 372 (1982) ; [Erratum-ibid. 185, 406.1988 APNYA,281,409 (1988 APNYA,281,409-449.2000)].

[6] S. Deser, R. Jackiw and S. Y. Pi, Acta Phys. Polon. B 36, 27 (2005)

[7] E. Ayon-Beato and M. Hassaine, Annals Phys. 317, 175 and the Einstein tensor yields

$$
\begin{aligned}
G_{\mu \nu}^{\prime}= & G_{\mu \nu}-\frac{\nabla_{\mu} \nabla_{\nu} \rho}{\rho}+\frac{(D-1)}{(D-2)} \frac{\nabla_{\mu} \rho \nabla_{\nu} \rho}{\rho^{2}} \\
& +g_{\mu \nu}\left[\frac{\square \rho}{\rho}-\frac{(D-1)}{2(D-2)} \frac{\nabla_{\sigma} \rho \nabla^{\sigma} \rho}{\rho^{2}}\right],
\end{aligned}
$$

or equivalently

$$
\begin{aligned}
G_{\mu \nu}=\quad & G_{\mu \nu}^{\prime}+\frac{\nabla_{\mu}^{\prime} \nabla_{\nu}^{\prime} \rho}{\rho}-\frac{D-3}{D-2} \frac{\nabla_{\mu} \rho \nabla_{\nu} \rho}{\rho^{2}} \\
& +g_{\mu \nu}^{\prime}\left[-\frac{\square^{\prime} \rho}{\rho}+\frac{(3 D-7)}{2(D-2)} \frac{g^{\prime \alpha \beta} \nabla_{\alpha} \rho \nabla_{\beta} \rho}{\rho^{2}}\right] .
\end{aligned}
$$

(2005); Phys. Rev. D 71, 084004 (2005).

[8] R. Jackiw, Weyl invariant dynamics in 3 dimensions, arXiv:gr-qc/0509035

[9] E. Ayon-Beato and M. Hassaine, Exploring AdS waves via nonminimal coupling, manuscript in preparation.

[10] G. W. Gibbons and D. A. Rasheed, Nucl. Phys. B 476, 515 (1996)

[11] G. W. Gibbons, Phantom matter and the cosmological constant, arXiv:hep-th/0302199

[12] Our conventions are the following: the signature is $(-+$ $+), \epsilon_{012}=+1$, the Riemann tensor is $R_{\mu \nu \rho}{ }^{\sigma}=+\partial_{\nu} \Gamma_{\mu \rho}^{\sigma}-$ $\ldots$ and the Ricci tensor is $R_{\mu \nu}=+\partial_{\alpha} \Gamma_{\mu \nu}^{\alpha}-\ldots$ 